\title{
Tres variedades de sarcoma de Kaposi con compromiso gastrointestinal
}

\section{Three varieties of Kaposi's sarcoma with gastrointestinal involvement}

\author{
Karina Jiménez, MD, ${ }^{1}$ Julián D. Martínez, MD, ${ }^{2}$ Geovanny Hernández, MD, ${ }^{3}$ Martín A. Garzón, MD, ${ }^{3}$ Natán Hormaza, MD, ${ }^{3}$ \\ Jorge Iván Lizarazo, MD, ${ }^{3}$ Juan Carlos Marulanda, MD, ${ }^{3}$ Juan Carlos Molano, MD, ${ }^{3}$ Mario H. Rey, MD, ${ }^{3}$ Martín Gómez Zuleta, MD. ${ }^{2}$
}

1 Residente, Medicina Interna, Universidad de la Sabana. Hospital Universitario de La Samaritana. Bogotá, Colombia

2 Facultad de Medicina, Universidad Nacional de Colombia. Bogotá, Colombia

3 Unidad de Gastroenterología, Hospital Universitario de La Samaritana. Bogotá, Colombia

Fecha recibido: $29-09-14$ Fecha aceptado: $21-07-15$

\begin{abstract}
Resumen
Se presentan casos de 4 pacientes adultos con 3 variedades de sarcoma de Kaposi: epidémico (asociado con infección por virus de inmunodeficiencia humana), iatrogénico (en paciente con inmunosupresión crónica y en un hombre con cirrosis alcohólica) y clásico (en paciente anciana sin inmunosupresión conocida); todos ellos presentaron compromiso gastrointestinal. Se hace una breve revisión de esta enfermedad.
\end{abstract}

\author{
Palabras clave \\ Sarcoma, Kaposi, compromiso, gastrointestinal.
}

\section{Abstract}

This article presents the cases of four adult patients with three varieties of Kaposi's sarcoma: epidemic, associated with human immunodeficiency virus infections; iatrogenic, associated with chronic immune suppression (and in this case in a man with alcoholic cirrhosis); and classic, occurring in elderly patient without previously known immunosuppression. All four cases had gastrointestinal involvement. A brief review of the disease is included.

Keywords

Kaposi's sarcoma, sarcoma, gastrointestinal compromiso.

\section{INTRODUCCIÓN}

El sarcoma de Kaposi (SK) es una neoplasia maligna compleja de origen mesenquimal, asociada con el herpes virus humano tipo $8(\mathrm{HV})$, que normalmente coincide con una alteración inmunológica que promueve un medio ambiente ideal para su desarrollo (1, 2). Existen 4 tipos de SK: el clásico, el endémico o africano (que tienen una distribución racial y geográfica específica, así como un curso más benigno), el asociado con VIH/ sida y el iatrogénico (hasta el momento han sido descritos con mayor asociación con inmunosupresión por trasplantes y tienen un peor pronóstico). La principal localización del SK es a nivel cutáneo, luego en los ganglios linfáticos, y en tercer lugar se encuentra el compromiso gastrointestinal, donde hay hallazgos endoscópicos variables que van desde úlceras hasta lesiones submucosas nodulares de color púrpura. En la histología, el principal hallazgo es la proliferación vascular caracterizada por la presencia de hendiduras vasculares, extravasación de hematíes con depósitos de hemosiderina, y la inmunohistoquímica positiva para herpes virus 8 (HV-8) (1-5).

\section{PRESENTACIÓN DE LOS CASOS}

Se presentan 4 pacientes con diagnóstico histológico de sarcoma de Kaposi, atendidos en la unidad de gastroenterología del Hospital Universitario de La Samaritana, Bogotá D.C, en los años de 2012 y 2013, con fines didácticos. Las características más importantes de los pacientes se resumen en la tabla 1 y luego se muestran los hallazgos macro y microscópicos de estos casos. 
Tabla 1. Características principales de los pacientes con sarcoma de Kaposi.

\begin{tabular}{|c|c|c|c|c|c|c|}
\hline Paciente & Sexo & Edad & $\begin{array}{l}\text { Motivo de } \\
\text { consulta }\end{array}$ & $\begin{array}{l}\text { Factor de } \\
\text { riesgo }\end{array}$ & Tratamiento & Localización \\
\hline 1 & Masculino & 42 & Úlcera en periné & VIH/sida & $\begin{array}{l}\text { TMP-SMX, abacavir, } \\
\text { lamivudina, efavirenz }\end{array}$ & $\begin{array}{l}\text { Estómago, colon } \\
\text { (figura 1) }\end{array}$ \\
\hline 2 & Masculino & 60 & $\begin{array}{l}\text { Hemoptisis, disnea, } \\
\text { anasarca }\end{array}$ & $\begin{array}{l}\text { Inmunosupresión crónica por } \\
\text { poliangeitis microscópica con } \\
\text { compromiso de pulmón, riñón }\end{array}$ & $\begin{array}{l}\text { Ciclofosfamida, azatioprina, } \\
\text { prednisolona }\end{array}$ & $\begin{array}{l}\text { Sistémico: boca, } \\
\text { pulmón, estómago, } \\
\text { duodeno (figuras 2-4) }\end{array}$ \\
\hline 3 & Masculino & 66 & $\begin{array}{l}\text { Hematemesis, } \\
\text { melenas }\end{array}$ & $\begin{array}{l}\text { Cirrosis alcohólica, síndrome } \\
\text { de hipertensión portal }\end{array}$ & $\begin{array}{l}\text { Propanolol, } \\
\text { espironolactona, lactulosa }\end{array}$ & Estómago (figura 5) \\
\hline 4 & Femenino & 89 & $\begin{array}{l}\text { Lesiones cutáneas } \\
\text { violáceas en piernas, } \\
\text { edema de MMII, } \\
\text { adenomegalia inguinal }\end{array}$ & $\begin{array}{l}\text { Edad avanzada, ninguno } \\
\text { demostrado }\end{array}$ & Ninguno & $\begin{array}{l}\text { Piel, estómago } \\
\text { (figura 6) }\end{array}$ \\
\hline
\end{tabular}

TMP-SMX: trimetoprim-sulfametoxazol; VIH: virus de inmunodeficiencia humana.

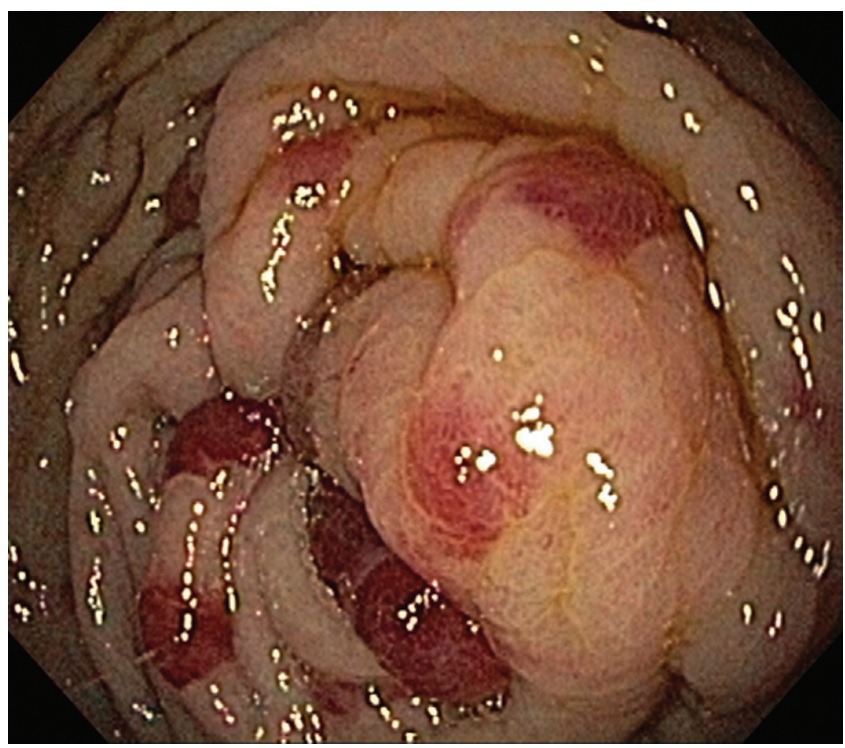

Figura 1. Visión endoscópica de lesiones numulares, violáceas de SK en estómago.

\section{DISCUSIÓN}

Esta enfermedad se reseñó por primera vez en 1872 por el dermatólogo húngaro Moritz Kaposi, que describió en 5 pacientes un "sarcoma idiopático, múltiple, pigmentado de la piel" con compromisos de los vasos sanguíneos y linfáticos, que luego fue denominado en su honor como sarcoma de Kaposi (SK) (6). Se han documentado varios tipos de SK: el clásico es diagnosticado en pacientes ancianos, generalmente descendientes de poblaciones mediterráneas o judíos de Europa del este, de crecimiento lento y

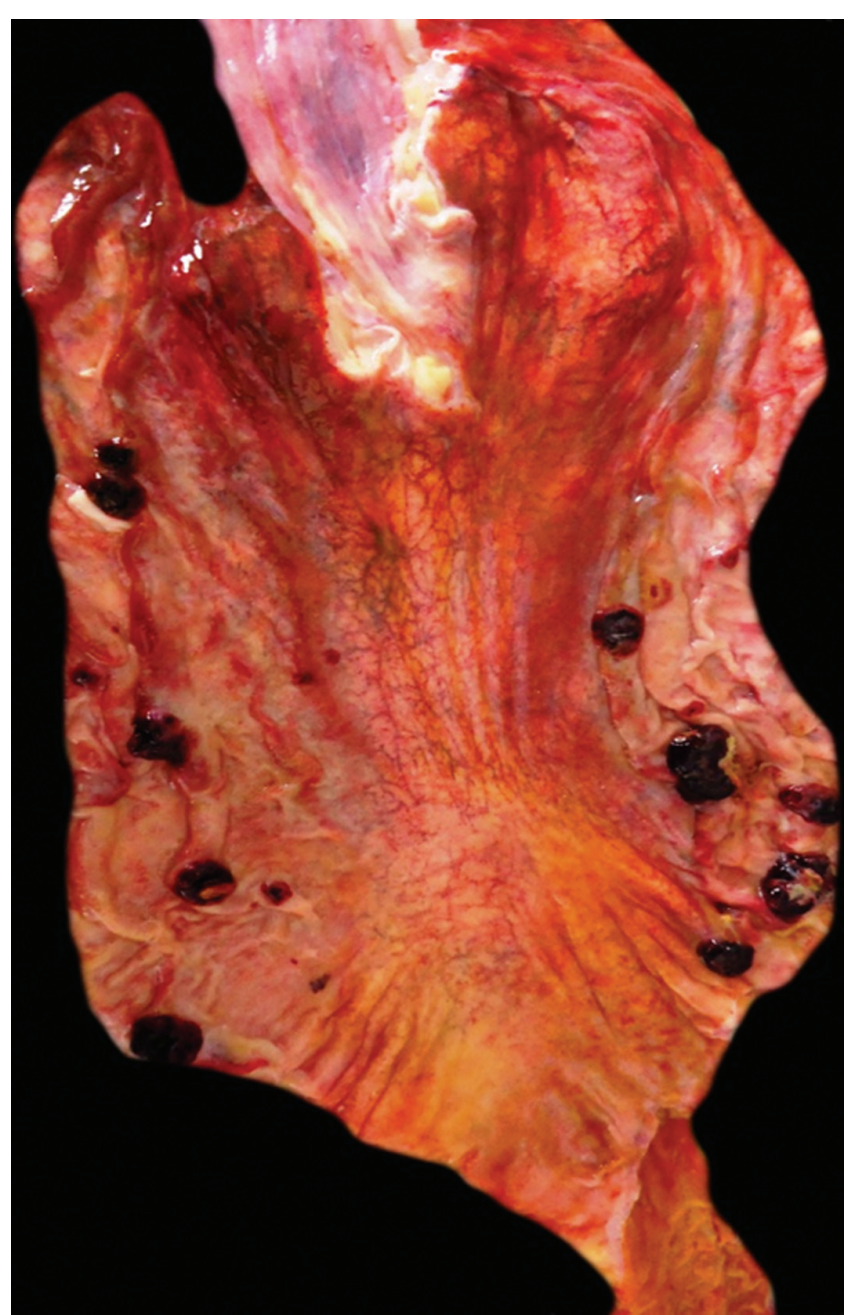

Figura 2. Pieza de autopsia, múltiples placas violáceas de SK en mucosa gástrica. 


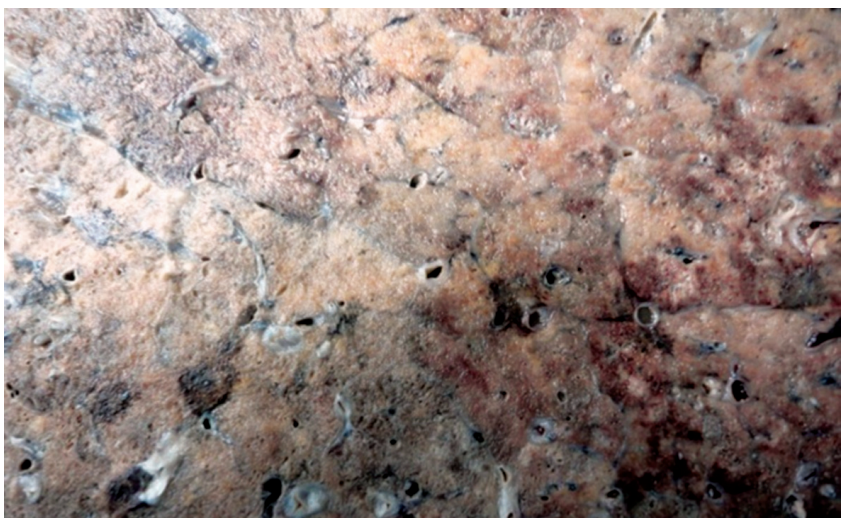

Figura 3. Pieza de autopsia, múltiples nódulos de SK pulmonares.

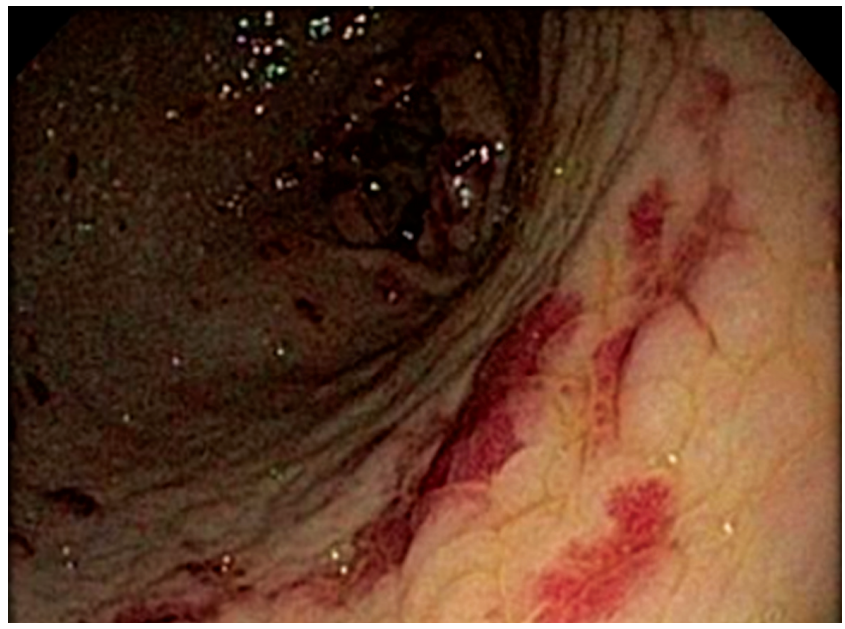

Figura 5. Visión endoscópica, placas violáceas de SK en estómago.

sus lesiones cutáneas afectan principalmente los miembros inferiores; el endémico, descrito en hombres preadolescentes y jóvenes de raza negra del África subsahariana; el epidémico o asociado con la infección por VIH/sida, con una proporción de afectación por géneros de 10 de hombres por 1 mujer, con mayor riesgo de desarrollarse en hombres homosexuales y con bajos recuentos de linfocitos CD4 y altas cargas virales; y la llamada forma iatrogénica o secundario de inmunosupresión farmacológica, especialmente asociado con enfermedades reumatológicas y trasplantes, con mayores reportes en los trasplantes renales. Aunque la histología en los diferentes tipos de SK es idéntica, los desenlaces son diferentes, siendo el asociado con el VIH/ sida el de peor pronóstico por su curso acelerado y generalmente fatal $(1,2,6)$.

El SK ha sido asociado con la infección por HV-8, el cual fue descrito en 1994, siendo luego asociado con SK (KS/HV, su sigla en inglés), y en el año 2010 fue declarado

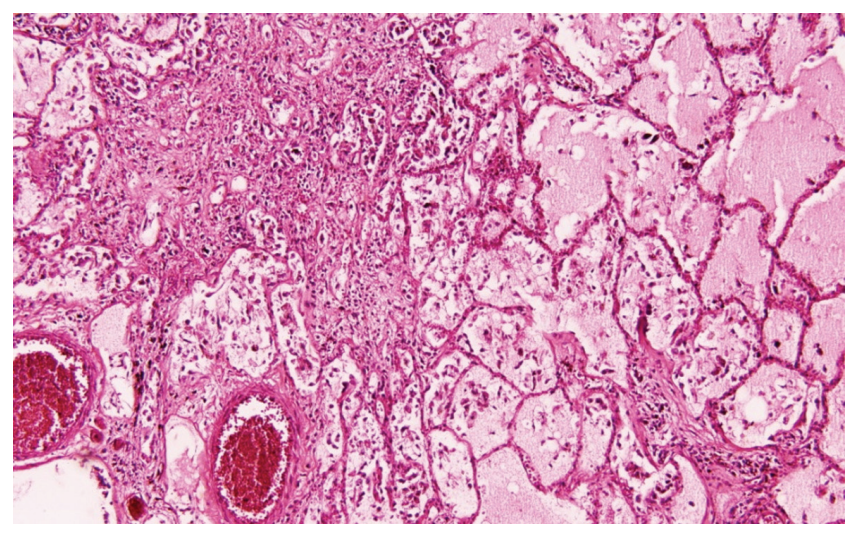

Figura 4. Histopatologia de sarcoma de Kaposi pulmonar.

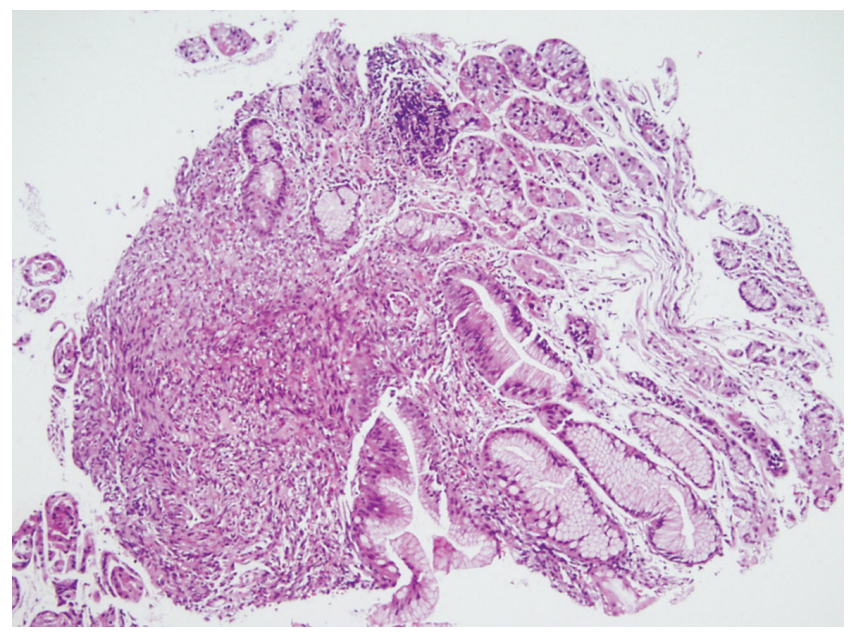

Figura 6. Histopatología de sarcoma de Kaposi gástrico.

como agente carcinogénico del grupo I por la Agencia Internacional para Estudio del Cáncer (7).

La prevalencia de la infección por KS/HV es variable en el mundo, con zonas endémicas asociadas con la epidemia del VIH/sida; sin embargo, tras la aparición de esta, el diagnóstico de SK ha tenido un incremento inicial dramático, con posterior estabilización tras la instauración de una terapia antirretroviral efectiva (8). La seroprevalencia global de infección por HV-8 no es bien conocida debido a la gran variabilidad interregional, sin embargo, se calcula que la incidencia de SK en pacientes con VIH/sida es 20000 veces mayor que en la población general y 300 veces más que en personas con otras causas de inmunosupresión (9). El SK representa el 60\% de los tumores diagnosticados en pacientes con VIH/sida $(2,8,9)$; para Colombia, la prevalencia estimada por el Sistema Nacional de Vigilancia en Salud Pública (SIVIGILA) de VIH/sida para el año 2012 fue de 122197 personas, pero se desconocen datos específi- 
cos a nivel nacional para el SK. Saldarriaga y colaboradores publicaron un estudio en el que se evaluaron pacientes con SK y VIH/sida entre 1962 y 2007, basados en el registro poblacional de cáncer en Cali y los datos de SIVIGILA; el período con mayor tasa de diagnóstico para hombres fue entre los años de 1996 y 2002, siendo de 4 por 100000 habitantes; después del año 2002 creció el diagnóstico de SK extracutáneo, que previamente era de solo el 10\% (10). No se dispone de información estadística con respecto al diagnóstico de SK iatrogénico en Colombia. Existen datos publicados en la literatura que indican que para el año 1985, el SK clásico correspondía al 1\% de las neoplasias en pacientes con una media de edad de 65 años (11). En Medellín, Orozco y colaboradores publicaron un estudio de la relación existente entre el HV-8 y el SK, encontrando que en el grupo de pacientes con SK y VIH/sida, el 83,3\% fueron positivos para anticuerpos contra $\mathrm{HV}-8$ por la técnica de inmunofluorescencia (12).

En la literatura se documentan con frecuencia casos de sarcoma de Kaposi asociado con VIH/sida, con predominio de manifestaciones de lesiones cutáneas o de las mucosas visibles (oral, faringe) sospechosas de SK y con posterior confirmación histopatológica (13), sin que exis$\tan$ recomendaciones internacionales sobre la búsqueda activa de la enfermedad sistémica. Cuando se encuentra compromiso gastrointestinal, generalmente es de forma incidental y no por sospecha directa de la existencia de esta patología, pues las indicaciones de realizar estudios endoscópicos al paciente inmunosuprimido no difieren del inmunocompetente, la diferencia radica en que más del $50 \%$ de los pacientes con $\mathrm{VIH} /$ sida presentan síntomas gastrointestinales y la etiología causal probablemente estará asociada con una infección oportunista $(2,13)$. El SK con compromiso gastrointestinal puede ser asintomático, sin embargo, puede presentarse con dolor abdominal, sangrado digestivo e incluso obstrucción intestinal, siendo tales síntomas la indicación para los estudios endoscópicos. Los hallazgos endoscópicos son variables: se pueden encontrar nódulos submucosos violáceos o rojos, placas de igual color o formaciones polipoides $(3,14)$.

El primer caso informado plantea la duda de si el paciente con diagnóstico de $\mathrm{VIH} /$ sida en estado avanzado y sin de síntomas gastrointestinales debiera ser llevado a estudio endoscópico de vías digestivas como prueba de tamización, dada la probabilidad que curse con SK gastrointestinal a pesar de no tener lesiones cutáneas, ya que se ha documentado que hasta el $15 \%$ de los pacientes con SK visceral no cursan con dicho compromiso (15).

En relación con el sarcoma de Kaposi iatrogénico, son pocos los casos publicados, la mayoría en relación con inmunosupresión farmacológica de enfermedades reumatológicas. En general, en la literatura se encuentran múlti- ples reportes de SK iatrogénico asociado con trasplantes; en la literatura inglesa se han informado pocos casos de pacientes inmunosuprimidos farmacológicamente de manera crónica con esteroides y azatioprina, cuyos diagnósticos de base corresponden a colitis ulcerativa, lupus eritematoso sistémico y vasculitis $(16,17)$. En la literatura médica de Iberoamérica reportada en la plataforma de Literatura Latinoamericana y del Caribe en Ciencias de la Salud (LILACS) se informan algunos casos asociados con inmunosupresión crónica por uso de esteroides y en pacientes con trasplantes renales $(18,19)$.

El segundo caso aquí reportado es poco común y de una magnitud inmensa que desencadenó la muerte del paciente, lo que sugiere considerar que los pacientes con inmunosupresión por otras causas diferentes a $\mathrm{VIH} /$ sida, y especialmente de origen medicamentoso asociado con enfermedades autoinmunes, son más susceptibles a la infección por HV-8 y su manifestación de SK, lo que implica una comorbilidad que empeora su pronóstico, y que es posible que su prevalencia aumente debido al incremento progresivo en el diagnóstico de enfermedades reumatológicas, en el uso temprano de terapias inmunosupresoras, y también en el aumento de los trasplantes de diversos órganos y la mayor sobrevida alcanzada por estos pacientes en nuestro país.

El tercer caso informado es un llamado de atención para considerar que las enfermedades sistémicas como la cirrosis y el alcoholismo, frecuentes en nuestro medio, conllevan a la alteración de la función inmune y a una mayor susceptibilidad a las infecciones virales y al desarrollo de neoplasias gastrointestinales como el SK, además de la mayor frecuencia de tumores escamosos de boca y esófago $\mathrm{y}$ adenocarcinomas gástricos y de páncreas, $\mathrm{y}$ a la necesidad de tomar biopsias de las lesiones focales gástricas encontradas en estos pacientes cuya semejanza endoscópica con otras lesiones vasculares (como la gastropatía por hipertensión portal y las ectasias vasculares antrales [watermelon stomach]) es muy grande $(3,5,13,14)$.

El último caso plantea la presencia de SK clásico en un paciente adulto mayor, con compromiso gastrointestinal y de miembros inferiores, con edema y adenopatías inguinales. Según lo reportado en la literatura, estos hallazgos acompañan las lesiones de extremidades inferiores en un $48 \%$ y $23 \%$, respectivamente (20). Lo anterior lleva a considerar este diagnóstico de SK gastrointestinal mediante la realización de estudios endoscópicos en pacientes ancianos con hallazgos cutáneos, que pueden beneficiarse de tratamientos oportunos con resultados positivos.

\section{REFERENCIAS}

1. Schwartz RA, Lamber WC. Kaposi sarcoma. Medicine Oncology [home page on the internet] [Updated: Apr 23, 
2009, cited 2010 Jun 20] [about 4 screens]. Available from: http://emedicine.medscape.com/e medicine $\backslash K a p o s i$ Sarcoma eMedicine.

2. Douglas Jl, Gustin JK, Moses A, Dezube BJ, Pantanowitz L. Kaposi sarcoma pathogenesis: A triad of viral infection, oncogenesis and chronic inflammation. Transl Biomed. 2010;14(7):172.

3. Martínez JD, Hernández G, Salinas C, Hormaza N, Garzón M, Lizarazo J, et al. Sarcoma de Kaposi gástrico en un paciente con virus de inmunodeficiencia humana (VIH): informe de caso. Rev Gastroenterol Perú. 2014;34:145-7.

4. Jiménez F, Barbaglia Y, Juárez L, Bucci P, Tedeschi F, Zalazar F. Sarcoma de Kaposi gastrointestinal asociado a síndrome de inmunodeficiencia adquirida. Descripción de un caso. Acta Gastroenterol Latinoam. 2011;41:308-11.

5. Taccogna S, Crescenzi A, Stasi R, Turrini L, Gallo A, Rossi Z. Kaposi sarcoma of the stomach: A case report. BMJ Case Rep. 2009: 2009.pii:bcr03.2009.1666.

6. Hengge UR, Ruzicka T, Tyring SK, et al. Update on Kaposi's sarcoma and other HHV8 associated diseases. Part 1: Epidemiology, environmental predispositions, clinical manifestations, and therapy. Lancet Infect Dis. 2002;2(5):281-92.

7. Chang Y, Cesarman E, Pessin MS, Lee F, Culpepper J, Knowles DM, et al. Identification of herpesvirus-like DNA sequences in AIDS-associated Kaposi's sarcoma. Science. 1994;266:1865-9.

8. Rohner E, Wyss N, Trelle S, Mbulaiteye SM, Egger M, Novak U, et al. HHV-8 seroprevalence: A global view. Syst Reviews. 2014;12(3):11.

9. Mohanna S, Maco V, Bravo F, Gotuzzo E, Bhaijee F, Subramony Ch, et al. Epidemiology and clinical characteristics of classic Kaposi's sarcoma, seroprevalence, and variants of human herpesvirus 8 in South America: A critical review of an old disease. Int J Infect Dis. 2005;9: 239-50.

10. Saldarriaga-Cantillo A, Bravo LE, Londoño O, García LS, Collazos P. Vigilancia epidemiológica del complejo VIH/ SIDA a través del análisis de tendencia de la incidencia del sarcoma de Kaposi. Colomb Med. 2012;43(4):273-80.
11. García A, Olivella F, Valderrama S, Rodríguez G. Kaposi's sarcoma in Colombia. Cancer. 1989;64:2393-8.

12. Orozco B, Gómez LM, Estrada S, Restrepo BN, Cataño JC. Relación entre el virus humano herpes 8 y el sarcoma de Kaposi en pacientes positivos y negativos para el VIH. Infectio. 2007;11(3):111-7.

13. Bhaijee F, Subramony Ch, Tang SJ, Pepper DJ. Human immunodeficiency virus-associated gastrointestinal disease: common endoscopic biopsy diagnoses. Pathol Res Int. 2011;2011:247923.

14. Sánchez del Monte J, Hernández-Guerrero A, SobrinoCossio S, Lárraga A, Sánchez-Benítez G, López Blanco P, et al. Manifestaciones clínicas y características endoscópicas del sarcoma de Kaposi en pacientes con síndrome de inmunodeficiencia adquirida. Rev Gastroenterol Mex. 2005;70(4):17-20.

15. Attia S, Dezube BJ, Torrealba JR, Sossman JM, Pau PR, et al. AIDS-related Kaposi's sarcoma of the gastrointestinal tract. J Clin Oncol. 2010;28(6):e250-1.

16. Jakob L, Metzler G, Chen KM, Garbe C. Non-AIDS associated Kaposi's sarcoma: Clinical features and treatment outcome. PLoS ONE. 2011;6(4):e18397.

17. Svrcek M, Tiret E, Bennis M, Guyot P, Fléjou JF. KSHV/ HHV8-associated intestinal Kaposi's sarcoma in patient with ulcertive colitis receiving immunosuppressive drugs: Report the case. Dis Colon Rectum. 2009;52(1):178-80.

18. Koche B, Hohmann C, Lima AC, Becker FL, Bonamigo R. Sarcoma de Kaposi associado à corticoterapia sistémica. Rev AMRIGS. 2010;54(2):202-5.

19. Jiménez E, Jiménez EE. Sarcoma de Kaposi en un adulto con transplante renal. Medisan. 2010;14(7):1000-4.

20. Bejar C, Ayaipoma A, Chián C. Casos clínicos, Sarcoma de Kaposi clásico. Folia Dermatol Perú. 2008;19 (1):27-31.

21. Hiatt KM, Nelson AM, LichyJM, Fanburg JC. Classic Kaposi sarcoma in the United States over the last two decades: A clinicopathologic and molecular study of 438 non-HIV-related Kaposi sarcoma patients with comparison to HIV-related Kaposi sarcoma. Mod Pathol. 2008;21(5):572-582. 